\title{
Impact of statins on risk of new onset diabetes mellitus: a population-based cohort study using the Korean National Health Insurance claims
} database

\author{
This article was published in the following Dove Press journal: \\ Therapeutics and Clinical Risk Management \\ II October 2016 \\ Number of times this article has been viewed
}

\author{
Jimin Lee' \\ Yoojin Noh' \\ Sooyoung Shin' \\ Hong-Seok Lim² \\ Rae Woong Park ${ }^{3}$ \\ Soo Kyung Bae ${ }^{4}$ \\ Euichaul $\mathrm{Oh}^{4}$ \\ Grace Juyun $\mathrm{Kim}^{5}$ \\ Ju Han Kim ${ }^{5}$ \\ Sukhyang Lee' \\ 'Division of Clinical Pharmacy, \\ College of Pharmacy, Ajou University, \\ Suwon, South Korea; ${ }^{2}$ Department \\ of Cardiology, School of Medicine, \\ Ajou University, Suwon, South \\ Korea; ${ }^{3}$ Department of Biomedical \\ Informatics, School of Medicine, \\ Ajou University, Suwon, South Korea; \\ ${ }^{4}$ Division of Pharmaceutical Sciences, \\ College of Pharmacy, The Catholic \\ University of Korea, Bucheon, South \\ Korea; ${ }^{5}$ Division of Biomedical \\ Informatics, College of Medicine, \\ Seoul National University, Seoul, \\ South Korea
}

Correspondence: Sukhyang Lee Department of Clinical Pharmacy, College of Pharmacy, Ajou University, 206 Worldcup-ro Yeongtong-gu, Suwon 443-749, South Korea

Tel +82312193443

Fax +82312193435

Email suklee@ajou.ac.kr

\begin{abstract}
Statin therapy is beneficial in reducing cardiovascular events and mortalities in patients with atherosclerotic cardiovascular diseases. Yet, there have been concerns of increased risk of diabetes with statin use. This study was aimed to evaluate the association between statins and new onset diabetes mellitus (NODM) in patients with ischemic heart disease (IHD) utilizing the Korean Health Insurance Review and Assessment Service claims database. Among adult patients with preexisting IHD, new statin users and matched nonstatin users were identified on a 1:1 ratio using proportionate stratified random sampling by sex and age. They were subsequently propensity score matched further with age and comorbidities to reduce the selection bias. Overall incidence rates, cumulative rates and hazard ratios (HRs) between statin use and occurrence of NODM were estimated. The subgroup analyses were performed according to sex, age groups, and the individual agents and intensities of statins. A total of 156,360 patients (94,370 in the statin users and 61,990 in the nonstatin users) were included in the analysis. The incidence rates of NODM were $7.8 \%$ and $4.8 \%$ in the statin users and nonstatin users, respectively. The risk of NODM was higher among statin users (crude HR 2.01, 95\% confidence interval [CI] 1.93-2.10; adjusted HR 1.84, 95\% CI 1.63-2.09). Pravastatin had the lowest risk (adjusted HR 1.54, 95\% CI 1.32-1.81) while those who were exposed to more than one statin were at the highest risk of NODM (adjusted HR 2.17, 95\% CI 1.93-2.37). It has been concluded that all statins are associated with the risk of NODM in patients with IHD, and it is believed that our study would contribute to a better understanding of statin and NODM association by analyzing statin use in the real-world setting. Periodic screening and monitoring for diabetes are warranted during prolonged statin therapy in patients with IHD.
\end{abstract}

Keywords: Atorvastatin, Fluvastatin, Lovastatin, Rosuvastatin, Pitavastatin, Pravastatin, Simvastatin, Ischemic heart disease, IHD, new onset diabetes mellitus, NODM

\section{Introduction}

In collaboration with the National Heart, Lung, and Blood Institute, the American College of Cardiology and the American Heart Association released updated guidelines for the treatment of blood cholesterol for primary and secondary reduction of atherosclerotic cardiovascular diseases. The Expert Panel identified specific patient groups who are most likely to benefit from statin therapy and recommended initiation of moderate- or high-intensity statin therapy based on the patient's risk profile. ${ }^{1}$

3-hydroxy-3-methylglutaryl-coenzyme A (HMG-CoA) reductase inhibitors, statins, are proven to reduce major cardiovascular outcomes, ${ }^{2-4}$ but there are concerns regarding 
the risk related to statin use. ${ }^{5}$ Clinical trials reported that statins reduced the risk of type II diabetes mellitus (T2DM) or were beneficial for reducing coronary events in people with T2DM. ${ }^{6,7}$ However, more recently, studies have raised concerns regarding the risk related to the use of statins. One of the most noticeable issues is that statin use may increase the risk of developing T2DM. ${ }^{1,8-10}$

T2DM affects $>300$ million individuals and contributes to significant morbidities and mortalities worldwide. ${ }^{11}$ T2DM has been recognized as an independent risk factor for ischemic heart disease (IHD), and evidence shows that in patients with established IHD, comorbidity of T2DM significantly increases IHD-related mortality rate. ${ }^{12}$ T2DM is increasing especially in Asian countries, and studies have shown that Asian individuals are at higher risk of developing T2DM than people of European ancestry. ${ }^{13}$

Nevertheless, only a small number of Asians were included in pivotal clinical trials, and clinical practice guidelines do not consider ethnicity in their recommendations for optimizing statin therapy in patients with cardiovascular diseases. ${ }^{1,8,14-16}$ Data suggest that Asian individuals are more sensitive to statin therapy and hence adverse effects may be greater. ${ }^{17,18}$ The overall effects of statin therapy on T2DM in Asian patients with IHD are largely unknown, and little attention has been given to possible differences among statin agents and intensities. Therefore, we utilized the Korean Health Insurance Review and Assessment Service (HIRA) claims database to evaluate the association between statin use and new onset diabetes mellitus (NODM) in patients with IHD.

\section{Materials and methods \\ Data source}

This was a retrospective cohort study conducted using the Korean HIRA database. The database consists of records, which health care institutions submit for medical claim reimbursement to the HIRA, of all the beneficiaries of the Korean National Health Insurance program. The National Health Insurance program is a universal health care system that allows beneficiaries to access any of the contracted medical facilities and institutions in Korea with low co-payment. ${ }^{19}$ Out of pocket costs apply to all enrollees for hospital and pharmacy visits. Those who are unable to afford co-payments are covered by the national insurance and exempted from copayments. Therefore, the HIRA database consists of records of all Koreans including the lowest socio-economic classes.

The database comprises clinic and hospital visit records that consist of patient information such as age, sex, diagnosis, medical procedures and services, type of health care institution, dates of clinic visits, admission dates, discharge dates, length of hospitalization, and medical specialty. Additionally, it consists of information regarding prescribed medications, such as brand and generic names, single administration doses, total daily doses, strength, route of administration, prescription date, and prescribed total day supply. The diagnoses were coded using the sixth revision of the Korean Standard Classification of Diseases, which reflects the tenth revision of the International Classification of Diseases. The medical procedures and services were coded according to the Current Procedural Terminology.

Prior to obtaining the data set, HIRA encrypted the original identification of each patient to protect patient privacy. The authors were also blinded to each patient's full personal identification number. The study obtained an official approval from the HIRA inquiry commission in replacement of the authors' institutional review board. The HIRA inquiry commission deemed patient consent not necessary.

\section{Patient population}

The aforementioned medical information were obtained for all adult patients ( $\geq 18$ years of age) diagnosed with IHD (Korean Standard Classification of Diseases: I20 - 25) between January 1, 2009 and December 31, 2012 (Figure 1A). First, patients who initiated statin therapy at any point during the index period (January 1, 2010 to June 30, 2010) as statin users were identified. The date of the first statin prescription record was considered the index date for the statin users. We deliberately limited the statin users to patients with no recent history of statin use by excluding patients with record of statin use in the year preceding their index date.

Patients were also excluded from the study if they were diagnosed with T2DM prior to the index date, if they had a history of antidiabetic medication use before the index date, if statins were initiated prior to or after the index period, if the length of statin therapy was $<12$ weeks, or if they used nonstatin cholesterol-lowering agents.

Patients who did not have a record of statin use in inpatient or ambulatory care claims at any time were considered as nonstatin users. The index date of the nonstatin users was the date of the first prescription record with no statin use that appeared in the HIRA database during the same index period (January 1, 2010 to June 30, 2010). Patients were also excluded if they were diagnosed with T2DM prior to the index date or had a history of antidiabetic medication use before the index date.

\section{Exposure to statins}

In Korea, during the index period, the commercially available statin products were atorvastatin, rosuvastatin, simvastatin, 


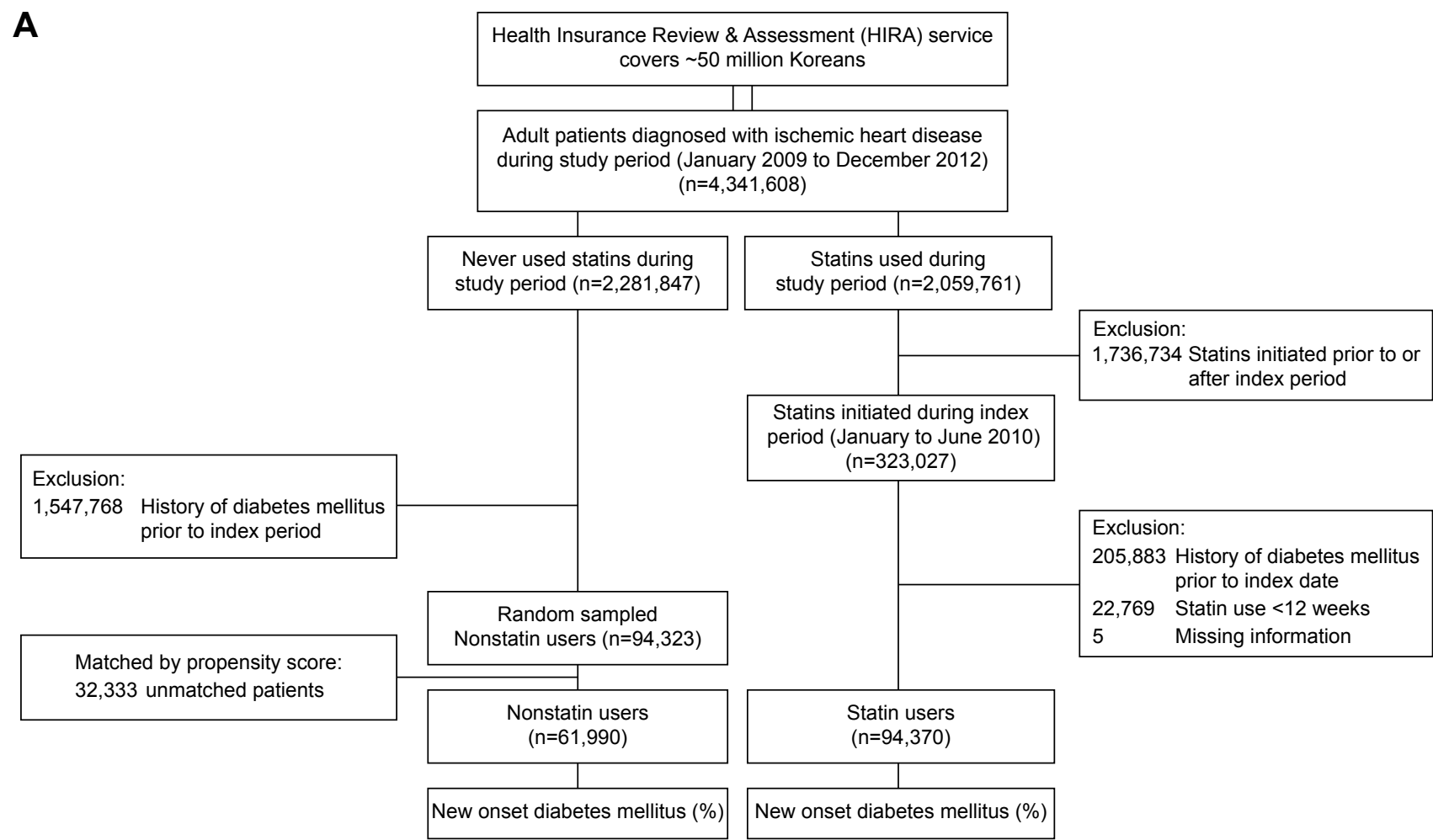

B

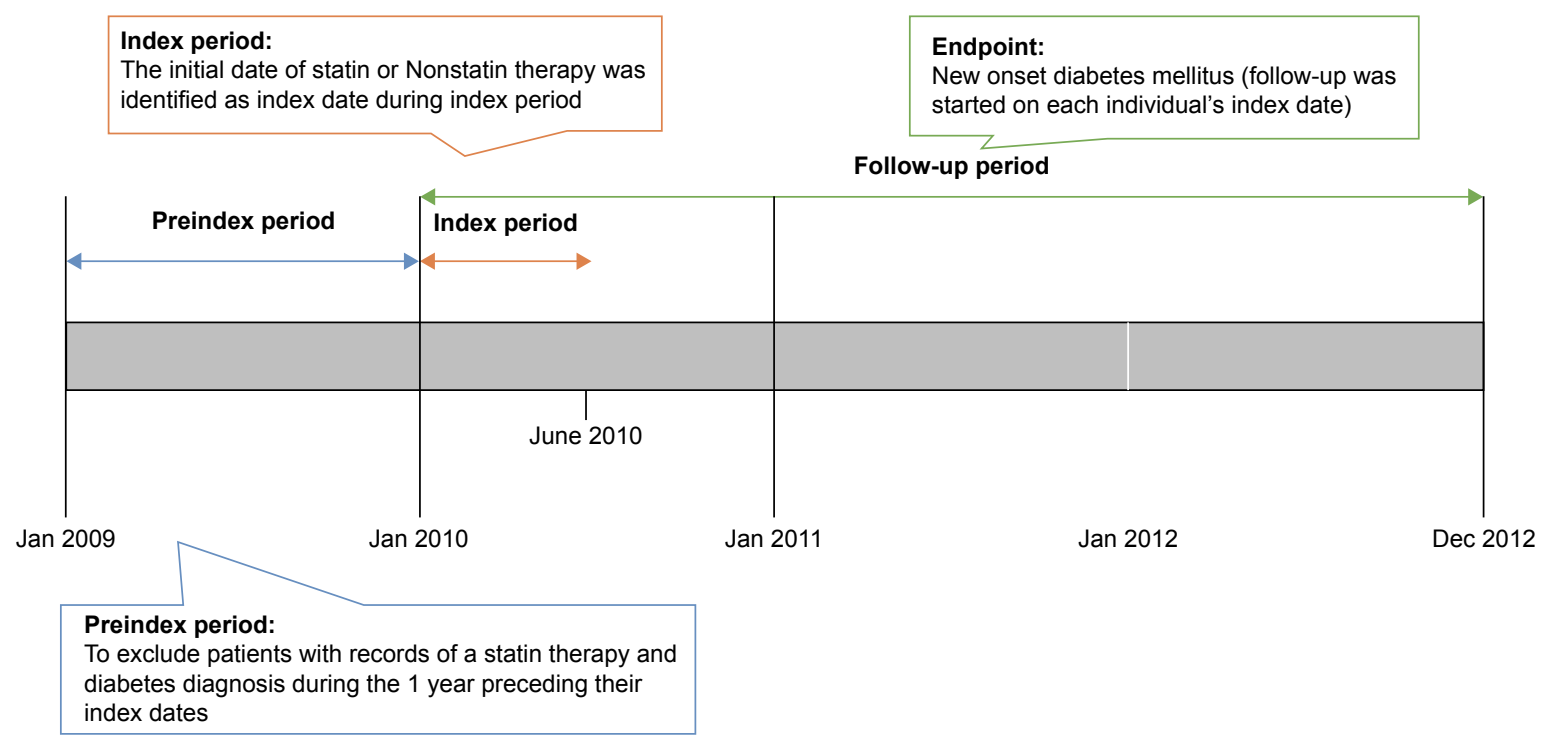

Figure I Flow chart of the study population and schematic description of the study design.

Notes: (A) Flowchart of the study population. (B) Schematic description of the study design.

pravastatin, lovastatin, fluvastatin, and pitavastatin. Patients who had used more than one statin agent, wherein therapy was switched from one statin to another, were classified into a complex group. The average daily dosage was calculated to determine the intensity of the therapy. Statin therapy intensities were assigned based on recommendations from the 2013 American College of Cardiology/American Heart Association guidelines. ${ }^{1}$

\section{Main outcome}

The primary outcome was NODM during statin therapy in previously statin-naïve patients with IHD (Figure 1B). Follow-up started from the index date, and all patients were followed up until they developed NODM, were censored from the database owing to death, showed no record of medical claims for more than a year, or until December 31, 2012, whichever came first. 
NODM was defined as a record of a T2DM diagnosis and prescription of one or more antidiabetic agents. The risk of NODM was expressed as cases per persons ( $\%$, NODM cases divided by the number of statin users or nonstatin users), and the NODM incidence rate was expressed as incidence rates per 100 person-years which were NODM cases divided by total person-time for the each group (Tables 1 and 2). The cumulative incidence of NODM of statin users versus nonstatin users during the follow-up period is described as number of events per 10,000 people (Figure 2).

\section{Covariates}

Covariates included sex, age, comorbidities such as hyperlipidemia, hypertension, heart failure, peripheral artery disease, unstable angina, cerebrovascular disease, chronic kidney disease, chronic liver disease, and history of myocardial infarction. These variables were considered possible confounders between statin use and risk of NODM.

\section{Statistical analysis}

Statin users and nonstatin users were matched by age and sex using proportionate stratified random sampling. A propensity score (PS) analysis was then carried out on sampled cohorts with logistic regression on the demographic and preindex characteristics, including age and comorbidities to address selection bias and the presence of potential confounding variables. Demographic and clinical characteristics between statin users and nonstatin users were compared using the chi-square test for categorical variables and the $t$-test for continuous variables. Analyses were adjusted for age, sex, statin agents, intensities, and comorbidities.

The cumulative rates of NODM during the follow-up period were estimated using Kaplan-Meier estimates of cumulative incidence ( 1 minus Kaplan-Meier estimator) and plotted according to time. The overall incidence rates and rates per 100 person-years were calculated and unadjusted and adjusted hazard ratios (HRs) were presented using a 95\% confidence interval (CI). Cox proportional hazard regression model was used to examine the association between the use of statin therapies and the occurrence of NODM. Subgroup analyses were also performed according to individual statin agents and intensities. All analyses were carried out with SAS statistical software (version 9.4. for Windows; SAS Institute, Inc., Cary, NC, USA).

\section{Results}

Of the $4,341,608$ adult IHD patients, 156,360 patients $(94,370$ statin users and 61,990 nonstatin users) were included in our analysis. The baseline characteristics of statin users and nonstatin users are presented in Table 3. At baseline, the mean (standard deviation) age was 60.84 (11.63) and 60.96 (11.92) years for statin users and nonstatin users, respectively, and $40.2 \%$ of the study population were $\geq 65$ years. Male patients represented $44.8 \%$ and $44.6 \%$ for statin users and nonstatin users, respectively. Statin users were more likely to have hyperlipidemia ( $40.2 \%$ vs $8.1 \%$ ), hypertension

Table I Incidence rates and HRs for NODM among statin users versus nonstatin users according to the individual statin agents and intensities

\begin{tabular}{|c|c|c|c|c|c|c|c|c|}
\hline Variables & $\begin{array}{l}\text { Patients } \\
(A, n)\end{array}$ & $\%$ & $\begin{array}{l}\text { NODM } \\
(B, n)\end{array}$ & Risk, $\frac{B}{A}(\%)$ & $\begin{array}{l}\text { Total } \\
\text { PYs }\end{array}$ & $\begin{array}{l}\text { Incidence } \\
\text { rate/I } 00 \text { PYs }\end{array}$ & $\begin{array}{l}\text { Crude HR } \\
(95 \% \mathrm{Cl})\end{array}$ & $\begin{array}{l}\text { Adjusted } \mathbf{H R}^{\mathrm{a}} \\
(95 \% \mathrm{Cl})\end{array}$ \\
\hline Non-statin users & 61,990 & 39.6 & 3,001 & 4.8 & 243,764 & 1.66 & I.00 (reference) & 1.00 (reference) \\
\hline Statin users & 94,370 & 60.4 & 7,383 & 7.8 & 195,042 & 3.79 & $2.01(1.93-2.10)$ & $1.84(1.63-2.09)$ \\
\hline Overall & 156,360 & 100.0 & 10,384 & 6.6 & 438,806 & 2.61 & - & - \\
\hline \multicolumn{9}{|l|}{ Statin agents } \\
\hline Atorvastatin & 58,036 & 61.5 & 4,634 & 8.0 & 120,729 & 3.84 & $2.04(1.95-2.14)$ & $2.05(1.96-2.16)$ \\
\hline Rosuvastatin & ||$, 85 \mid$ & 12.6 & 957 & 8.1 & 25,400 & 3.77 & $2.00(1.86-2.16)$ & $2.00(1.85-2.15)$ \\
\hline Simvastatin & 13,012 & 13.8 & 1,002 & 7.7 & 25,346 & 3.95 & $2.10(1.96-2.26)$ & $2.12(1.97-2.28)$ \\
\hline Pravastatin & 2,733 & 2.9 & 163 & 6.0 & 5,652 & 2.88 & $1.53(1.31-1.80)$ & $1.54(1.32-1.81)$ \\
\hline Lovastatin & 833 & 0.9 & 64 & 7.7 & I,589 & 4.03 & $2.14(1.67-2.74)$ & $2.16(1.68-2.77)$ \\
\hline Fluvastatin & 1,262 & 1.3 & 77 & 6.1 & $2,47 \mid$ & 3.12 & $1.65(1.32-2.07)$ & $1.66(1.32-2.08)$ \\
\hline Pitavastatin & 4,075 & 4.3 & 279 & 6.8 & 8,789 & 3.17 & 1.69 (1.49-1.91) & $1.70(1.50-1.92)$ \\
\hline Complex & 2,568 & 2.7 & 207 & 8.1 & 5,066 & 4.09 & $2.18(1.89-2.5 I)$ & $2.17(1.93-2.37)$ \\
\hline Statin users, total & 94,370 & 100.0 & 7,383 & 7.8 & 195,042 & 3.79 & 2.01 (1.93-2.10) & $1.84(1.63-2.09)$ \\
\hline \multicolumn{9}{|l|}{ Intensity } \\
\hline Low & 3,796 & 4.0 & 127 & 6.2 & 4,030 & 3.15 & $1.67(1.40-2.00)$ & $1.69(1.4 I-2.01)$ \\
\hline Moderate & 88,529 & 93.8 & 6,961 & 7.9 & 183,077 & 3.80 & $2.02(1.94-2.11)$ & $2.03(1.94-2.12)$ \\
\hline High & 2,045 & 2.2 & 295 & 7.8 & 7,935 & 3.72 & $1.98(1.75-2.23)$ & $1.97(1.74-2.22)$ \\
\hline Statin users, total & 94,370 & 100.0 & 7,383 & 7.8 & 195,042 & 3.79 & 2.01 (1.93-2.10) & $1.84(1.63-2.09)$ \\
\hline
\end{tabular}

Notes: 'Adjusted HR was calculated using the Cox proportional hazard model adjusting for the comorbidities. "-” Indicates not applicable.

Abbreviations: $\mathrm{Cl}$, confidence interval; HR, hazard ratio; NODM, new onset diabetes mellitus; PY, person-year. 


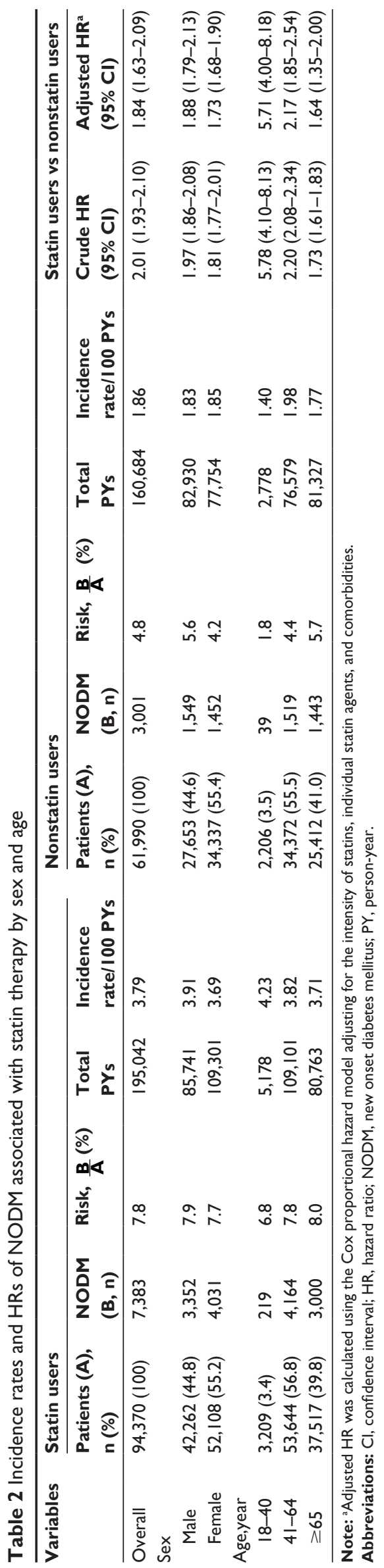

( $57.8 \%$ vs $41.9 \%)$, heart failure (3.1\% vs $2.5 \%)$, history of myocardial infarction ( $2.3 \%$ vs $0.7 \%$ ), and cerebrovascular disease (11.6\% vs $7.3 \%)$ than nonstatin users. Of the statin users, $61.5 \%$ took atorvastatin, $12.6 \%$ rosuvastatin, $13.8 \%$ simvastatin, $4.3 \%$ pitavastatin, $2.9 \%$ pravastatin, and $2.7 \%$ complex therapy (Table 1). A total of $93.8 \%$ users took moderate-intensity statins, whereas $4.0 \%$ and $2.2 \%$ took high- and low-intensity statins, respectively (Table 1).

A total of 10,384 out of 156,360 patients experienced NODM, including 7,383 (7.8\%) of 94,370 statin users and $3,001(4.8 \%)$ of 61,990 nonstatin users. The crude and covariate-adjusted HRs for the primary endpoint, in statin users versus nonstatin users, were 2.01 (95\% CI 1.93-2.10) and 1.84 (95\% CI 1.63-2.09), respectively (Table 1).

The overall incidence of NODM in statin users was higher than in nonstatin users (3.79 vs 1.86 per 100 personyears) (Table 1). Compared to nonstatin users, the adjusted NODM HRs were 1.69 (95\% CI 1.41-2.01), 2.03 (95\% CI 1.94-2.12), and 1.97 (95\% CI 1.74-2.22) for the lowmoderate, and high-intensity statin users, respectively. Among the statin agents, pravastatin had the lowest risk of NODM (adjusted HR 1.54, 95\% CI 1.32-1.81) while those who were exposed to more than one type of statin, the socalled complex group, were at the highest risk of NODM (adjusted HR 2.18, 95\% CI 1.89-2.51). The Kaplan-Meier estimates demonstrate that cumulative rates of NODM during the follow-up period were higher in statin users compared with nonstatin users, which correlated with the crude and adjusted HRs ( $P$-value $<0.0001$, Figure $2 A$ ).

Sex-specific analysis showed that the adjusted NODM HR for statin users versus nonstatin users was more significant among male patients (adjusted HR 1.88, 95\% CI 1.79-2.13) (Table 2); the incidence of NODM was the most frequent among male statin users (Figure 2B). Age-specific analysis showed that the risk of NODM was most significant among statin users under the age of 40 years (adjusted HR 5.71, 95\% CI 4.00-8.18) (Figure 2C and Table 2).

In addition, there was a significant difference between the groups with respect to the time to NODM; average time to NODM was 329.9 days in the statin users and 465.5 days in the nonstatin users.

Baseline characteristics and preliminary results of the study subjects prior to PS matching can be found in the supplemental document (Tables S1 and S2).

\section{Discussion}

This study, undertaken using a large-scale database that contains the medical information of $\sim 50$ million Korean individuals, showed that statin therapy is associated with an 
A

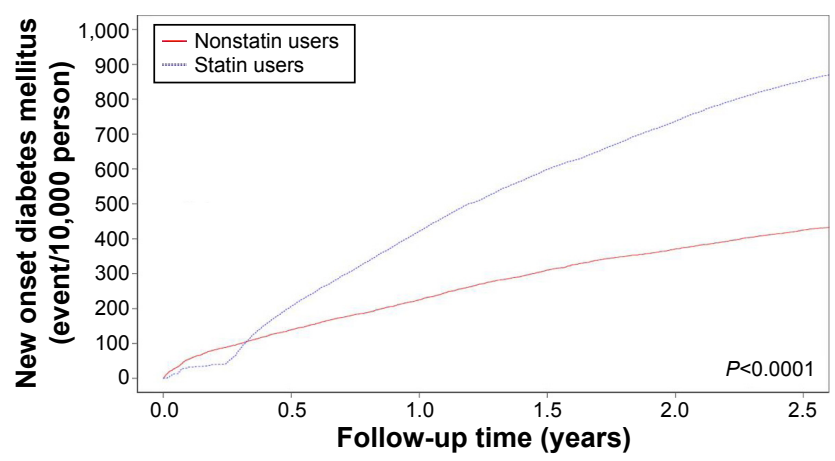

B

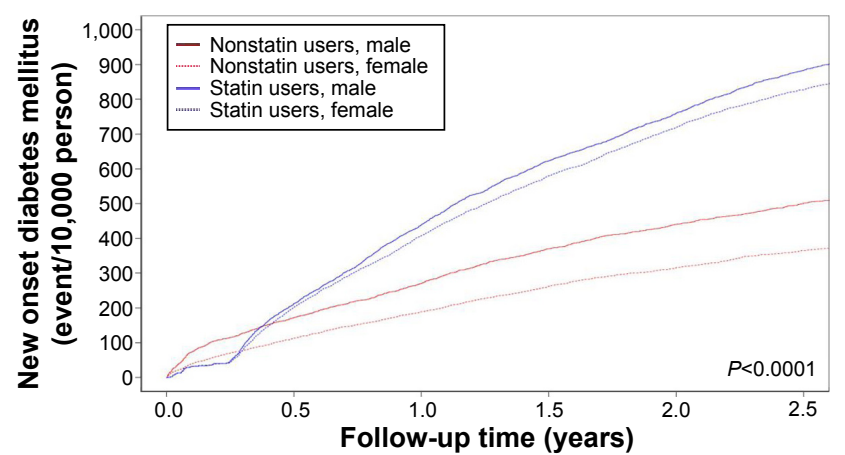

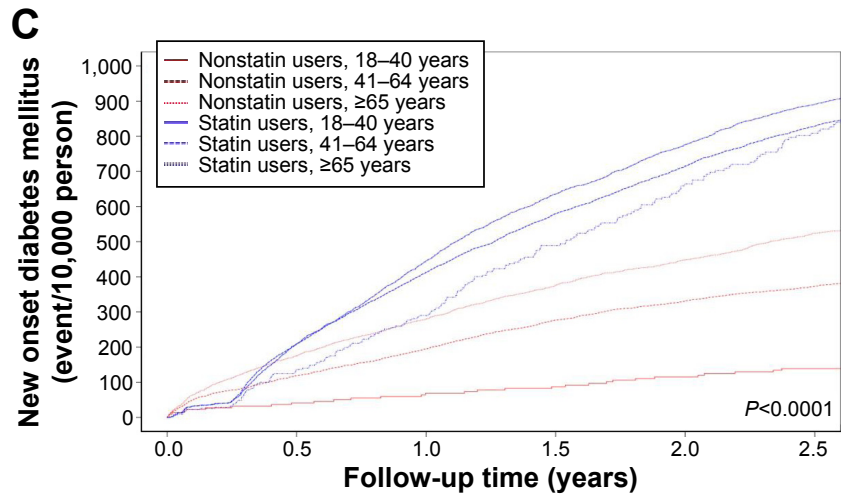

Figure 2 Cumulative incidence of NODM between statin users and nonstatin users during the follow-up period.

Notes: (A) Statin users versus nonstatin users. (B) Statin users versus nonstatin users, divided by sex. (C) Statin users versus nonstatin users, divided by age groups. Abbreviation: NODM, new onset diabetes mellitus.

increased risk of NODM in patients with IHD. In addition, all statins were associated with NODM risk.

The Justification for the Use of Statins in Primary Prevention: An Intervention Trial Evaluating Rosuvastatin (JUPITER) study was the first placebo-controlled clinical trial to raise the issue of a potential increased risk of NODM in patients using statins. Ridker et al reported that rosuvastatin modestly increases the risk of developing NODM in patients with no history of CVD, as cases of newly diagnosed T2DM were more frequent in the rosuvastatin group (270 [3.0\%] cases in the statin group vs $216[2.4 \%]$ in the placebo group, $P=0.01){ }^{8}$

Table 3 Baseline characteristics of study subjects $(n=156,360)$

\begin{tabular}{|c|c|c|c|c|c|}
\hline \multirow[t]{2}{*}{ Characteristics } & \multicolumn{2}{|c|}{ Statin users $n=94,370$} & \multicolumn{2}{|c|}{ Nonstatin users $n=61,990$} & \multirow[t]{2}{*}{$P$-value } \\
\hline & $\mathbf{N}$ & $\%$ & $\mathbf{N}$ & $\%$ & \\
\hline Sex (male) & 42,262 & 44.8 & 27,653 & 44.6 & 0.497 \\
\hline Age, years (mean $\pm S D)$ & $60.84 \pm 11.63$ & & $60.96 \pm 11.92$ & & $<0.000$ I \\
\hline Age, years (median, IQR) & $61.0(53.0-69.0)$ & & $61.0(52.0-70.0)$ & & $<0.000$ I \\
\hline $18-40$ & 321 & 3.4 & 2,206 & 3.5 & $<0.000 \mathrm{I}$ \\
\hline $4 I-64$ & 53,644 & 56.8 & 34,372 & 55.5 & \\
\hline$\geq 65$ & 37,517 & 39.8 & 25,412 & 41.0 & \\
\hline \multicolumn{6}{|l|}{ Comorbidities } \\
\hline Hyperlipidemia & 37,924 & 40.2 & 5,029 & 8.1 & $<0.000$ I \\
\hline Hypertension & 54,574 & 57.8 & 25,960 & 41.9 & $<0.0001$ \\
\hline Heart failure & 3,039 & 3.1 & 1,526 & 2.5 & $<0.000$ I \\
\hline PAD & 9,634 & 10.2 & 4,699 & 7.6 & $<0.000 \mathrm{I}$ \\
\hline History of MI & 2,122 & 2.3 & 409 & 0.7 & $<0.0001$ \\
\hline UA & 3,415 & 3.6 & 1,212 & 2.0 & $<0.000$ I \\
\hline CVD & 10,962 & 11.6 & 4,545 & 7.3 & $<0.0001$ \\
\hline CKD (stage I-V) & 771 & 0.8 & 449 & 0.7 & 0.04 \\
\hline CLD & 400 & 0.4 & 169 & 0.3 & 0.19 \\
\hline
\end{tabular}

Abbreviations: CKD, chronic kidney disease; CLD, chronic liver disease; CVD, cerebrovascular disease; IQR, interquartile range; MI, myocardial infarction; PAD, peripheral artery disease; SD, standard deviation; UA, unstable angina. 
Subsequent meta-analyses confirmed the observed effect. ${ }^{20,21}$ Rajpathak et al examined the effect of statins on T2DM risk by conducting a meta-analysis on six primary and secondary prevention trials, totaling 57,593 patients. Compared to the placebo group, those who received statin therapy had a $13 \%$ higher risk of T2DM (relative risk $1.13,95 \%$ CI $1.03-1.23) .{ }^{20}$ Results of a meta-analysis of 13 randomized controlled trials of statins with 91,140 patients showed the same trends; statin therapy was associated with a $9 \%$ increased risk of incident diabetes (odds ratio [OR] 1.09, $95 \%$ CI 1.02-1.17). ${ }^{21}$

Our results followed a trend similar to that of previous studies, ${ }^{8,20,21}$ however, the effect of statin-induced NODM appears to be much greater in our study population (adjusted HR 1.84, 95\% CI 1.63-2.09). The adverse effects of statins seem dose related. ${ }^{5,22,23}$ In addition, it could be attributed to the intensity of statins since the majority was treated with moderate- to high-intensity statins according to the American College of Cardiology/American Heart Association guideline recommendations.

Our results demonstrated that moderate-intensity therapy is most likely to be associated with NODM risk (adjusted HR of low-, moderate-, and high-intensity groups were 1.69 [1.41-2.01], 2.03 [1.94-2.12], and 1.97 [1.74-2.22]); however, previous studies suggested that NODM risk seemed intensity dependent. ${ }^{10,24}$ In a study of 136,936 patients hospitalized for a recent cardiovascular event or procedure, it was revealed that high-potency statins were associated with significantly higher NODM risk compared to lower-potency agents (rate ratio $1.15,95 \%$ CI $1.05-1.26$ ). ${ }^{10}$ The definition of high potency statins described in this study correlates with our definition of moderate-intensity therapy. Thus, this result is particularly relevant to our findings since the study design and the definition of the NODM endpoint are comparable to those of our study. The reason that high-intensity statins seem to have a lower risk of NODM than moderate intensity is likely because a very small number of high-intensity statin users were included in the analysis and this may not be enough to represent all the high-intensity statin users. Future studies are warranted to verify whether NODM risk is truly intensity dependent.

As shown in Figure 2C, among the statin users patients aged $\leq 40$ years are most likely to develop NODM and the risk appears to be lower with older age groups. This observation is in line with the findings of a recent study evaluating differential impact of statins on NODM in different age groups in Taiwanese women. ${ }^{25}$ The study was limited to female patients aged $\geq 40$ years; however, the result is worth noting that statin-related NODM was more evident in younger age groups (40-54 and 55-64 years: adjusted OR 1 [reference] and 0.86 [0.72-1.02], respectively) compared with older age groups (65-74 and $\geq 75$ years: adjusted OR 0.72 [0.59-0.87] and 0.63 [0.49-0.80], respectively). As mentioned in this study, the impact of age on statin-associated NODM is unclear and controversial. The finding seems counterintuitive since it is reasonable to assume that older statin users are at higher risk of NODM since well-known T2DM risk factors including inactivity, hypertension, and hyperlipidemia are more common in this population. The results are inconclusive in this regard, and therefore, further investigation is encouraged to explain the true impact of age on statin-associated NODM.

Recent data examined the risk of NODM among patients treated with different statin agents. Pravastatin as a reference drug, the risk of NODM was higher with simvastatin (adjusted HR 1.10, 95\% CI 1.04-1.17), rosuvastatin (adjusted HR 1.18, 95\% CI 1.10-1.26), and atorvastatin (adjusted HR $1.22,95 \%$ CI 1.15-1.29) while other statin agents showed no more increased risk of NODM. ${ }^{26}$ On the other hand, the effects of different statin agents were compared using no statin therapy as a reference. All types of statin therapies were associated with an increased risk of NODM, and pravastatin was associated with the lowest risk of NODM (adjusted HR 1.54, 95\% CI 1.32-1.81). However, associations between statin types and degree of NODM risk were inconsistent with previous studies. ${ }^{25,27,28}$ According to our findings, switching between different statins increases the risk of NODM. Additionally, lovastatin, simvastatin, atorvastatin, and rosuvastatin were associated with over twofold increased risk of NODM, while risks of other statin agents were somewhat lower. We understand that the study populations differ among the studies, which may attribute to these different findings. Patients' underlying conditions could potentially explain these controversies, and thus, larger controlled trials are warranted to investigate the association.

As with many established pharmacologic treatments, interethnic variability in the response to statin therapy has been reported. ${ }^{17,29}$ A pharmacokinetic study analyzing plasma exposure to statins revealed that Asian patients' statin plasma concentrations were nearly twofold higher than White subjects living in the same environment. ${ }^{17}$ It suggests that Asian individuals may be more prone to adverse effect. Our observations are in line with the findings of a recent study analyzing the association between moderateintensity statin and NODM among hospitalized patients in Korea (OR 1.99, 95\% CI 1.00-3.98).$^{30}$ Unlike the aforementioned studies where the majority of the patients were non-Asians, ${ }^{3,8,31-35}$ studies composed primarily of Asians 
show stronger statin-induced T2DM effects including the present study.

Not only that Asians are more sensitive to statins which predisposes them to a higher risk of NODM, studies have proposed that intensive therapy is no more effective in reducing major coronary events than moderate to low-dose statin therapies. ${ }^{36,37}$ Given these facts, it is postulated that Asian individuals may not require the guideline-recommended statin therapy to achieve clinical benefits. Low-intensity statins may be sufficient to adequately prevent IHD complications in Asian patients, while minimizing the risk of NODM.

Several possible mechanisms have been suggested to explain the diabetogenic effects of statins. One plausible hypothesis is that statins cause T2DM by altering glucose homeostasis through both impairment of insulin secretion and diminished insulin sensitivity. ${ }^{38}$ Statins modify glucose metabolism by reducing glucose uptake into skeletal muscles and adipose tissues secondary to downregulation of glucose transporters including insulin-sensitive solute carrier family 2, member 4 (SLC2A4, formerly known as GLUT4). ${ }^{39}$ Decreased expression of SLC2A4 likely contributes to insulin resistance and consequently causes T2DM. Studies have shown that atorvastatin and simvastatin decrease the expression of SLC2A4 in adipocytes and insulin sensitivity, which could potentially affect the onset of T2DM. ${ }^{39,40}$

In addition, statin-induced mitochondrial dysfunction in pancreatic beta-cells, skeletal cells, and adipocytes may lead to an impairment of insulin sensitivity and adiponectin secretion. ${ }^{41,42}$ In pancreatic beta-cells, mitochondria play a critical role in linking glucose metabolism with insulin exocytosis; thus, defects in mitochondrial function block this metabolic coupling and cause beta-cell death. ${ }^{41}$ Functional defects in adipocytes are linked to the dysregulation of glucose homeostasis and to insulin insensitivity. ${ }^{42}$

The last potential mechanism of statin-induced T2DM involves an adiponectin, insulin-sensitizing, and antiinflammatory cytokines released from adipocytes. Insulin sensitivity was reduced as a result of decreased plasma concentration of adiponectin in lipophilic statins including rosuvastatin, atorvastatin, and simvastatin whereas it increased in pravastatin-treated patients. ${ }^{43,44}$ These results support our findings that NODM risk with rosuvastatin, atorvastatin, and simvastatin is more significant than that with pravastatin.

It appears that statin-induced NODM is more than just an intensity-dependent effect. Although it is not fully understood, lipophilicity of statins seems to have an influence on statin-induced NODM as well. Our results showed that statin-induced NODM risk is apparent in the real-world data, and the effect may be more significant in Asian individuals with IHD. Further studies, especially prospective designed studies, are warranted to validate our findings.

Our study has some advantages since the national health insurance claims database was used, which contains information about nearly all the Koreans with IHD. Therefore, the results may be extrapolated to Asian individuals in general, having similar clinical conditions. Nonetheless, there are limitations to the present study. First, owing to the retrospective nature of the study, we were unable to verify whether the newly diagnosed diabetic patients had major risk factors for T2DM such as metabolic syndrome, impaired fasting glucose, increased body mass index, or elevated hemoglobin A1C. Additionally, the HIRA database does not contain information on other factors that could have influenced the analysis, such as height, weight, and family and social history, which would have permitted a more systematic evaluation of individuals. Second, validity of the claims data is limited. We had to rely on diagnoses recorded by treating physicians using the sixth revision of the Korean Standard Classification of Diseases, and thus the accuracy of recording was not verified. We overcame this limitation by strictly limiting NODM to those who had received a T2DM diagnosis on two separate occasions and who were prescribed anti-diabetic medications. This ensured that the statins' adverse effect was not overestimated. Lastly, because data were provided by a third party service, our data access was limited to the record of sampled cohort at the time of PS matching. Due to this limitation, PS matching was performed on the sampled cohort. We may have been able to have a more balanced cohort if PS matching was performed on the initial patient population.

Based on the current evidence, it is concluded that all statin therapies are associated with an increased risk of NODM in patients with IHD. Our finding is consistent with results from recent studies, ${ }^{10,21,24,45}$ although the adverse effect appears to be greater in our population. It is believed that our study contributes to a better understanding of the association between statins and NODM through the analysis of a real-world statin users. Especially in those aged $\leq 40$ years, the risk of statin-associated NODM seems to be greater. Before initiating statin therapy in these patients, lifestyle modification should be emphasized and statin's potential benefits versus adverse effects need to be discussed. In addition, periodic screening and monitoring for T2DM may be warranted in all patients with IHD undergoing statin therapy. 


\section{Acknowledgments}

This research was supported by the Bio \& Medical Technology Development Program of the National Research Foundation funded by the Ministry of Science, ICT \& Future Planning, Republic of Korea (No 2013M3A9B5075838). Special thanks to the Editage for the English editing of this manuscript.

\section{Disclosure}

The authors report no conflicts of interest in this work.

\section{References}

1. Stone NJ, Robinson JG, Lichtenstein AH, et al. 2013 ACC/AHA guideline on the treatment of blood cholesterol to reduce atherosclerotic cardiovascular risk in adults: a report of the American College of Cardiology/American Heart Association Task Force on Practice Guidelines. J Am Coll Cardiol. 2014;63(25 Pt B):2889-2934.

2. Sacks FM, Tonkin AM, Shepherd J, et al. Effect of pravastatin on coronary disease events in subgroups defined by coronary risk factors: the Prospective Pravastatin Pooling Project. Circulation. 2000;102(16): 1893-1900.

3. Shepherd J, Blauw GJ, Murphy MB, et al. Pravastatin in elderly individuals at risk of vascular disease (PROSPER): a randomised controlled trial. Lancet. 2002;360(9346):1623-1630.

4. Heart Protection Study Collaborative G. MRC/BHF Heart Protection Study of cholesterol lowering with simvastatin in 20,536 high-risk individuals: a randomised placebo-controlled trial. Lancet. 2002; 360(9326):7-22.

5. Bitzur R, Cohen H, Kamari Y, Harats D. Intolerance to statins: mechanisms and management. Diabetes Care. 2013;36(Suppl 2):S325-S330.

6. Freeman DJ, Norrie J, Sattar N, et al. Pravastatin and the development of diabetes mellitus: evidence for a protective treatment effect in the West of Scotland Coronary Prevention Study. Circulation. 2001;103(3) 357-362.

7. Collins R, Armitage J, Parish S, Sleigh P, Peto R; Heart Protection Study Collaborative G. MRC/BHF Heart Protection Study of cholesterollowering with simvastatin in 5963 people with diabetes: a randomised placebo-controlled trial. Lancet. 2003;361(9374):2005-2016.

8. Ridker PM, Danielson E, Fonseca FA, et al. Rosuvastatin to prevent vascular events in men and women with elevated C-reactive protein. N Engl J Med. 2008;359(21):2195-2207.

9. Culver AL, Ockene IS, Balasubramanian R, et al. Statin use and risk of diabetes mellitus in postmenopausal women in the Women's Health Initiative. Arch Intern Med. 2012;172(2):144-152.

10. Dormuth CR, Filion KB, Paterson JM, et al. Higher potency statins and the risk of new diabetes: multicentre, observational study of administrative databases. BMJ. 2014;348:g3244.

11. Sherwin R, Jastreboff AM. Year in diabetes 2012: the diabetes tsunami. J Clin Endocrinol Metab. 2012;97(12):4293-4301.

12. Grundy SM, Benjamin IJ, Burke GL, et al. Diabetes and cardiovascular disease: a statement for healthcare professionals from the American Heart Association. Circulation. 1999;100(10):1134-1146.

13. Chan JC, Malik V, Jia W, et al. Diabetes in Asia: epidemiology, risk factors, and pathophysiology. JAMA. 2009;301(20):2129-2140.

14. Fihn SD, Blankenship JC, Alexander KP, et al. 2014 ACC/AHA/AATS/ PCNA/SCAI/STS focused update of the guideline for the diagnosis and management of patients with stable ischemic heart disease: a report of the American College of Cardiology/American Heart Association Task Force on Practice Guidelines, and the American Association for Thoracic Surgery, Preventive Cardiovascular Nurses Association, Society for Cardiovascular Angiography and Interventions, and Society of Thoracic Surgeons. J Am Coll Cardiol. 2014;64(18):1929-1949.
15. Downs JR, Clearfield M, Weis S, et al. Primary prevention of acute coronary events with lovastatin in men and women with average cholesterol levels: results of AFCAPS/TexCAPS. Air Force/Texas Coronary Atherosclerosis Prevention Study. JAMA. 1998;279(20):1615-1622.

16. Cannon $\mathrm{CP}$, Braunwald $\mathrm{E}, \mathrm{McCabe} \mathrm{CH}$, et al. Intensive versus moderate lipid lowering with statins after acute coronary syndromes. $N$ Engl J Med. 2004;350(15):1495-1504.

17. Lee E, Ryan S, Birmingham B, et al. Rosuvastatin pharmacokinetics and pharmacogenetics in white and Asian subjects residing in the same environment. Clin Pharmacol Ther. 2005;78(4):330-341.

18. Kwon JE, Kim Y, Hyun S, et al. Cholesterol Lowering Effects of Lowdose Statins in Korean Patients. J Lipid Atheroscler. 2014;3(1):21-28.

19. Kwon S. Payment system reform for health care providers in Korea. Health Policy Plan. 2003;18(1):84-92.

20. Rajpathak SN, Kumbhani DJ, Crandall J, Barzilai N, Alderman M, Ridker PM. Statin therapy and risk of developing type 2 diabetes: a meta-analysis. Diabetes Care. 2009;32(10):1924-1929.

21. Sattar N, Preiss D, Murray HM, et al. Statins and risk of incident diabetes: a collaborative meta-analysis of randomised statin trials. Lancet. 2010;375(9716):735-742.

22. Golomb BA, Evans MA. Statin adverse effects: a review of the literature and evidence for a mitochondrial mechanism. Am J Cardiovasc Drugs. 2008;8(6):373-418.

23. Cham S, Evans MA, Denenberg JO, Golomb BA. Statin-associated muscle-related adverse effects: a case series of 354 patients. Pharmacotherapy. 2010;30(6):541-553.

24. Preiss D, Seshasai SR, Welsh P, et al. Risk of incident diabetes with intensive-dose compared with moderate-dose statin therapy: a metaanalysis. JAMA. 2011;305(24):2556-2564.

25. Chen CW, Chen TC, Huang KY, Chou P, Chen PF, Lee CC. Differential impact of statin on new-onset diabetes in different age groups: a population-based case-control study in women from an asian country. PLoS One. 2013;8(8): 71817

26. Carter AA, Gomes T, Camacho X, Juurlink DN, Shah BR, Mamdani MM. Risk of incident diabetes among patients treated with statins: population based study. BMJ. 2013;346:f2610.

27. Ma T, Chang MH, Tien L, Liou YS, Jong GP. The long-term effect of statins on the risk of new-onset diabetes mellitus in elderly Taiwanese patients with hypertension and dyslipidaemia: a retrospective longitudinal cohort study. Drugs Aging. 2012;29(1):45-51.

28. Ma T, Tien L, Fang CL, Liou YS, Jong GP. Statins and new-onset diabetes: a retrospective longitudinal cohort study. Clin Ther. 2012;34(9): 1977-1983.

29. Mabuchi H, Kita T, Matsuzaki M, et al. Large scale cohort study of the relationship between serum cholesterol concentration and coronary events with low-dose simvastatin therapy in Japanese patients with hypercholesterolemia and coronary heart disease: secondary prevention cohort study of the Japan Lipid Intervention Trial (J-LIT). Circ J. 2002;66(12):1096-1100.

30. Park JY, Rha SW, Choi B, et al. Impact of low dose atorvastatin on development of new-onset diabetes mellitus in Asian population: threeyear clinical outcomes. Int J Cardiol. 2015;184:502-506.

31. Kjekshus J, Apetrei E, Barrios V, et al. Rosuvastatin in older patients with systolic heart failure. N Engl J Med. 2007;357(22):2248-2261.

32. Sever PS, Dahlof B, Poulter NR, et al. Prevention of coronary and stroke events with atorvastatin in hypertensive patients who have average or lower-than-average cholesterol concentrations, in the Anglo-Scandinavian Cardiac Outcomes Trial - Lipid Lowering Arm (ASCOT-LLA): a multicentre randomised controlled trial. Lancet. 2003;361(9364):1149-1158.

33. Keech A, Colquhoun D, Best J, et al. Secondary prevention of cardiovascular events with long-term pravastatin in patients with diabetes or impaired fasting glucose: results from the LIPID trial. Diabetes Care. 2003;26(10):2713-2721.

34. Scandinavian Simvastatin Survival Study Group. Randomised trial of cholesterol lowering in 4444 patients with coronary heart disease: the Scandinavian Simvastatin Survival Study (4S). Lancet. 1994; 344:1383-1389. 
35. The ALLHAT Officers and Coordinators for the ALLHAT Collaborative Research Group. Major outcomes in moderately hypercholesterolemic, hypertensive patients randomized to pravastatin vs usual care: The Antihypertensive and Lipid-Lowering Treatment to Prevent Heart Attack Trial (ALLHAT-LLT). JAMA. 2002;288:2998-3007.

36. Armitage J, Bowman L, Wallendszus K, et al; Study of the Effectiveness of Additional Reductions in Cholesterol and Homocysteine (SEARCH) Collaborative Group. Intensive lowering of LDL cholesterol with $80 \mathrm{mg}$ versus $20 \mathrm{mg}$ simvastatin daily in 12,064 survivors of myocardial infarction: a double-blind randomised trial. Lancet. 2010;376(9753):1658-1669.

37. Pedersen TR, Faergeman O, Kastelein JJ, et al. High-dose atorvastatin vs usual-dose simvastatin for secondary prevention after myocardial infarction: the IDEAL study: a randomized controlled trial. JAMA. 2005;294(19):2437-2445.

38. Aiman U, Najmi A, Khan RA. Statin induced diabetes and its clinical implications. J Pharmacol Pharmacother. 2014;5(3):181-185.

39. Nakata M, Nagasaka S, Kusaka I, Matsuoka H, Ishibashi S, Yada T. Effects of statins on the adipocyte maturation and expression of glucose transporter 4 (SLC2A4): implications in glycaemic control. Diabetologia. 2006;49(8):1881-1892.
40. Ganesan S, Ito MK. Coenzyme Q10 ameliorates the reduction in GLUT4 transporter expression induced by simvastatin in 3T3-L1 adipocytes. Metab Syndr Relat Disord. 2013;11(4):251-255.

41. Supale S, Li N, Brun T, Maechler P. Mitochondrial dysfunction in pancreatic beta cells. Trends Endocrinol Metab. 2012;23(9):477-487.

42. Wang CH, Wang CC, Huang HC, Wei YH. Mitochondrial dysfunction leads to impairment of insulin sensitivity and adiponectin secretion in adipocytes. FEBS J. 2013;280(4):1039-1050.

43. Koh KK, Quon MJ, Han SH, et al. Differential metabolic effects of pravastatin and simvastatin in hypercholesterolemic patients. Atherosclerosis. 2009;204(2):483-490.

44. Koh KK, Sakuma I, Quon MJ. Differential metabolic effects of distinct statins. Atherosclerosis. 2011;215(1):1-8.

45. Park ZH, Juska A, Dyakov D, Patel RV. Statin-associated incident diabetes: a literature review. Consult Pharm. 2014;29(5):317-334. 


\section{Supplementary materials}

Table SI Baseline characteristics of study subjects prior to propensity score matching $(n=188,693)$

\begin{tabular}{|c|c|c|c|c|c|}
\hline \multirow[t]{2}{*}{ Variables } & \multicolumn{2}{|c|}{ Statin users, $n=94,370$} & \multicolumn{2}{|c|}{ Nonstatin users, $n=94,323$} & \multirow[t]{2}{*}{$P$-value } \\
\hline & $\mathbf{N}$ & $\%$ & $\mathbf{N}$ & $\%$ & \\
\hline Sex (male) & 42,262 & 44.8 & 42,237 & 44.8 & 0.99 \\
\hline Age, years (mean $\pm S D$ ) & $60.84 \pm 11.63$ & & $56.98 \pm 16.11$ & & $<0.000$ I \\
\hline Age, years (median, IQR) & $61.0(53.0-69.0)$ & & $58.0(46.0-70.0)$ & & $<0.0001$ \\
\hline $18-64$ & 56,853 & 60.2 & 57,470 & 60.9 & 0.0027 \\
\hline $65-74$ & 25,634 & 27.2 & 25,383 & 26.9 & \\
\hline$\geq 75$ & 11,883 & 12.6 & $\mathrm{II}, 470$ & 12.2 & \\
\hline \multicolumn{6}{|l|}{ Comorbidities } \\
\hline Hyperlipidemia & 37,924 & 40.2 & 5,037 & 5.3 & $<0.000$ I \\
\hline Hypertension & 54,574 & 57.8 & 34,129 & 36.2 & $<0.000$ I \\
\hline Heart failure & 3,039 & 3.1 & $2,|2|$ & 2.3 & $<0.000$ I \\
\hline PAD & 9,634 & 10.2 & 6,200 & 6.6 & $<0.000$ I \\
\hline History of Ml & 2,122 & 2.3 & 553 & 0.6 & $<0.000$ I \\
\hline UA & 3,415 & 3.6 & $\mathrm{I}, 570$ & 1.7 & $<0.000$ I \\
\hline CVD & 10,962 & 11.6 & 6,086 & 6.5 & $<0.000$ I \\
\hline CKD (stage I-V) & 771 & 0.8 & 600 & 0.65 & $<0.000$ I \\
\hline CLD & 400 & 0.4 & 234 & 0.3 & $<0.000$ I \\
\hline
\end{tabular}

Abbreviations: CKD, chronic kidney disease; CLD, chronic liver disease; CVD, Cerebrovascular disease; MI, myocardial infarction; PAD, peripheral artery disease; $\mathrm{SD}$, standard deviation; UA, unstable angina; IQR, interquartile range.

Table S2 Incidence rates and HR for NODM among statin users versus nonstatin users according to the individual statin agents and intensities prior to propensity score matching $(n=188,693)$

\begin{tabular}{|c|c|c|c|c|c|c|c|c|}
\hline & $\begin{array}{l}\text { Patients } \\
(A, n)\end{array}$ & $\%$ & $\begin{array}{l}\text { NODM } \\
(B, n)\end{array}$ & Risk, $\frac{B}{A}(\%)$ & $\begin{array}{l}\text { Total } \\
\text { PYs }\end{array}$ & $\begin{array}{l}\text { Incidence } \\
\text { rate// } 00 \text { PYs }\end{array}$ & $\begin{array}{l}\text { Crude HR } \\
(95 \% \mathrm{Cl})\end{array}$ & $\begin{array}{l}\text { Adjusted } \mathbf{H R}^{\mathrm{a}} \\
(95 \% \mathrm{Cl})\end{array}$ \\
\hline Non-statin users & 94,323 & 50.0 & 4,049 & 4.3 & 243,764 & 1.66 & $1.00[$ Reference $]$ & $1.00[$ Reference] \\
\hline Statin users & 94,370 & 50.0 & 7,383 & 7.8 & 195,042 & 3.79 & $2.27(2.19-2.36)$ & $1.83(1.53-2.18)$ \\
\hline Overall & 188,693 & 100.0 & 11,432 & 6.1 & 438,806 & 2.61 & - & - \\
\hline \multicolumn{9}{|l|}{ Statin agents } \\
\hline Atorvastatin & 58,036 & 61.5 & 4,634 & 8.0 & 120,729 & 3.84 & $2.31(2.2 \mathrm{I}-2.40)$ & $2.22(2.12-2.33)$ \\
\hline Rosuvastatin & $|I, 85|$ & 12.6 & 957 & 8.1 & 25,400 & 3.77 & $2.33(2.1 \mathrm{I}-2.43)$ & $2.20(2.04-2.36)$ \\
\hline Simvastatin & 13,012 & 13.8 & 1,002 & 7.7 & 25,346 & 3.95 & $2.37(2.21-2.54)$ & $2.28(2.12-2.45)$ \\
\hline Pravastatin & 2,733 & 2.9 & 163 & 6.0 & 5,652 & 2.88 & $1.73(1.48-2.02)$ & $1.67(1.43-1.96)$ \\
\hline Lovastatin & 833 & 0.9 & 64 & 7.7 & I,589 & 4.03 & $2.41(1.89-3.09)$ & $2.36(1.84-3.02)$ \\
\hline Fluvastatin & 1,262 & 1.3 & 77 & 6.1 & $2,47 \mid$ & 3.12 & $1.87(1.49-2.34)$ & 1.81 (1.45-2.27) \\
\hline Pitavastatin & 4,075 & 4.3 & 279 & 6.8 & 8,789 & 3.17 & $1.91(1.69-2.15)$ & $1.83(1.62-2.07)$ \\
\hline Complex & 2,568 & 2.7 & 207 & 8.1 & 5,066 & 4.09 & $2.46(2.14-2.83)$ & $2.47(2.15-2.85)$ \\
\hline Statin users, total & 94,370 & 100.0 & 7,383 & 7.8 & 195,042 & 3.79 & $2.27(2.19-2.36)$ & $1.83(1.53-2.18)$ \\
\hline \multicolumn{9}{|l|}{ Intensity } \\
\hline Low & 3,796 & 4.0 & 127 & 3.3 & 4,030 & 3.15 & $1.89(1.58-2.25)$ & $1.82(1.53-2.18)$ \\
\hline Moderate & 88,529 & 93.8 & 6,961 & 7.9 & 183,077 & 3.80 & $2.18(2.10-2.37)$ & $2.11(2.01-2.30)$ \\
\hline High & 2,045 & 2.2 & 295 & 14.4 & 7,935 & 3.72 & $2.23(I .98-2.5 I)$ & $2.22(1.93-2.46)$ \\
\hline Statin users, total & 94,370 & 100.0 & 7,383 & 7.8 & 195,042 & 3.79 & $2.27(2.19-2.36)$ & $1.83(1.53-2.18)$ \\
\hline
\end{tabular}

Notes: aAdjusted HR was calculated using the Cox proportional hazard model adjusting for the comorbidities. "-" Indicates not applicable.

Abbreviations: $\mathrm{Cl}$, confidence interval; $\mathrm{HR}$, hazard ratio; NODM, new onset diabetes mellitus; PY, person-year.

Therapeutics and Clinical Risk Management

\section{Publish your work in this journal}

Therapeutics and Clinical Risk Management is an international, peerreviewed journal of clinical therapeutics and risk management, focusing on concise rapid reporting of clinical studies in all therapeutic areas, outcomes, safety, and programs for the effective, safe, and sustained use of medicines. This journal is indexed on PubMed Central, CAS,

\section{Dovepress}

EMBase, Scopus and the Elsevier Bibliographic databases. The manuscript management system is completely online and includes a very quick and fair peer-review system, which is all easy to use. Visit http://www.dovepress.com/testimonials.php to read real quotes from published authors. 